\title{
THE POLAR ORLICZ-BRUNN-MINKOWSKI INEQUALITIES
}

\section{LIJUAN LIU}

Abstract. In this paper, we establish some Orlicz-Brunn-Minkowski type inequalities for (dual) quermassintegrals of polar bodies and star dual bodies, respectively.

Mathematics subject classification (2010): 52A20, 52A40.

Keywords and phrases: Convex body, Orlicz addition, radial Orlicz addition, Orlicz-Brunn-Minkowski inequality.

\section{REFERENCES}

[1] J. FIREY, Polar means of convex bodies and a dual to the Brunn-Minkowski theorem, Canad. J. Math., 13 (1961), 444-453.

[2] J. FIREY, p-means of convex bodies, Math. Scand., 10 (1962), 17-24.

[3] R. J. GARDNer, The Brunn-Minkowski inequality, Bull. Amer. Math. Soc., 39 (2002), 355-405.

[4] R. J. GARDNER, Geometric tomography, second edition, Encyclopedia of Mathematics and its Applications, 58, Cambridge University Press, Cambridge, 2006.

[5] R. J. Gardner, D. Hug And W. WeIL, The Orlicz Brunn-Minkowski theory: a general framework, additions, and inequalities, J. Differential Geom., 97 (2014), 427-476.

[6] R. J. Gardner, D. Hug, W. Weil And D. Ye, The dual Orlicz-Brunn-Minkowski theory, J. Math. Anal. Appl., 430 (2015), 810-829.

[7] G. H. Hardy, J. E. Littlewood and G. Pólya, Inequalities, Cambridge Univ. Press, London, 1934.

[8] C. Haberl, E. Lutwak, D. Yang and G. Zhang, The even Orlicz, Minkowski problem, Adv. Math., 224 (2010), 2485-2510.

[9] M. A. Hernández Cifre AND J. Y. Nicolás, On Brunn-Minkowski-type inequalities for polar bodies, J. Geom Anal., 26 (2014), 1-13.

[10] E. LutwaK, Dual mixed volumes, Pacific J. Math., 58 (1975), 531-538.

[11] E. LutwaK, The Brunn-Minkowski-Firey theory I: Mixed volumes and the Minkowski problem, J. Differential Geom., 38 (1993), 131-150.

[12] E. LuTwaK, The Brunn-Minkowski-Firey theory II: Affine and geominimal surface areas, Adv. Math., 118 (1996), 244-294.

[13] E. Lutwak, D. Yang And G. Zhang, Orlicz projection bodies, Adv. Math., 223 (2010), 220-242.

[14] E. Lutwak, D. Yang And G. Zhang, Orlicz centroid bodies, J. Differential Geom., 84 (2010), 365-387.

[15] M. MoszyŃska, Quotient star bodies, intersection bodies and star duality, J. Math. Anal. Appl., 232 (1999), 45-60.

[16] M. Moszyńs Ka, Selected Topics in Convex Geometry, Springer Verlag, 2005.

[17] C. Saroglou, More on logarithmic sums of convex bodies, Mathematika, 62 (2014), 818-841.

[18] R. SCHNEIDER, Convex bodies: the Brunn-Minkowski theory, second expanded edition, Encyclopedia of Mathematics and its Applications, Cambridge University Press, Cambridge, 2014.

[19] Y. WANG AND Q. HUANG, Orlicz-Brunn-Minkowski inequality for polar bodies and dual star bodies, Math. Inequal. Appl., 20 (2017), 1139-1144.

[20] D. XI, H. Jin And G. Leng, The Orlicz Brunn-Minkowski inequality, Adv. Math., 260 (2014), 350 374.

[21] G. Xiong And D. Zou, Orlicz mixed quermassintegrals, Sci. China Math., 57 (2014), 2549-2562. 
[22] B. Zhu, J. Zhou AND W. Xu, Dual Orlicz-Brunn-Minkowski theory, Adv. Math., 264 (2014), 700 725. 